\title{
Recent advances in optical bistability
}

\author{
from Y.R. Shen
}

IN recent years, optical bistability has been a subject of intense research in quantum electronics because of its potential usefulness in optical data processing and alloptical logic and computing systems ${ }^{1}$. There has already been a conference on the topic $^{2}$, and at the XIIth International Quantum Electronics Conference held in Munich in June, two sessions were devoted to the subject.

In a system with optical bistability, the relationship of output to input is characterized by hysteresis loops; at a given input, the steady-state output can be either high or low depending on the operation path. There bistable characteristics are the basis of a binary switching element. Indeed, it was earlier predicted and demonstrated that an optical Fabry-Perot (F-P) interferometer filled with a nonlinear medium should exhibit bistability. The principle of the device, including the transient ${ }^{3}$ and steady-state behaviour ${ }^{1}$ and the analogy with the phenomenon of phase transition ${ }^{4}$, is already fairly well understood. The more recent advances on the subject are mainly in materials and technology.

A most significant advance in the survey of materials for nonlinear F-P interferometers is the discovery of the superiority of the $\mathrm{GaAs}-\mathrm{GaAlAs}$ superlattice structure ${ }^{5}$. The future applications of optical bistability will require the development of small $(\sim 1 \mu \mathrm{m})$ fast $(\sim \mathrm{ps})$ low-operating-power $(\sim 1 \mu \mathrm{W})$ room-temperature devices. Semiconductors with an excitonic - a positronium-like entity in the solid - line transition appear to be most promising because of their marked nonlinearity and the very low power required to achieve saturation in absorption.

Optical bistability has indeed been demonstrated in a $4.1 \mu \mathrm{m}$ etalon of pure $\mathrm{GaAs}$ with an input laser beam at the exciton frequency ${ }^{6}$. Unfortunately, excitons in GaAs dissociate above $120 \mathrm{~K}$, while at low temperatures, the bistable switching time is long $(\sim \mu \mathrm{s})$. But recently it has been found that GaAs-Ga $\mathrm{Al}_{1-x} \mathrm{As}$ superlattices grown by molecular beam epitaxy can have a larger exciton-binding energy than that of pure GaAs because of the quantum-well effect. As a result, excitons can exist in a superlattice even at room temperature. The power required for saturation of the exciton transition at room temperature is also three times less than that for pure GaAs.

These features point towards the possible construction of a small efficient optical bistable device. Indeed, on a $2 \mu \mathrm{m}$ thick etalon of GaAs (335 $\AA$ )-Ga $\mathrm{Ga}_{0.73}$ $\mathrm{Al}_{0.27} \mathrm{As}(401 \AA)$ superlattice at room temperature, optical bistability can be observed with an $\sim 100 \mathrm{~mW}$ laser at the exciton frequency. The switching time is around $20-40$ ns. Further improvement can probably be made by using thinner layers in the superlattice structure.

Y.R. Shen is at the Department of Physics, University of California, Berkeley, and the Materials and Molecular Research Division, Lawrence Berkeley Laboratory, Berkeley, California 94720.

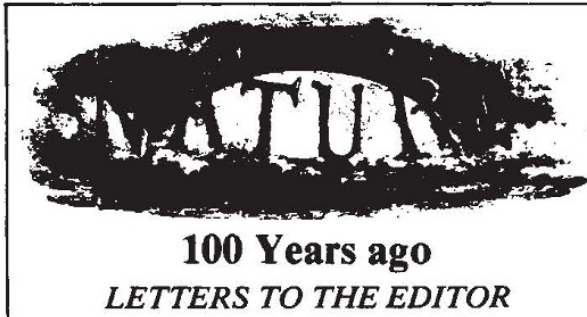

The Editor does not hold himself responsible for opinions expressed by his correspondents. Neither can he undertake 10 return or to correspond with the writers of, rejected manuscripts. No notice is taken of anonymous communication

The Editor urgently requests correspondents to keep their letters as short as possible. The pressure on his space is so great that it is impossible otherwise to ensure the appearance even of communications containing interesting and novel facts.

\section{The Comet}

I ENCLOSE a drawing made this morning after a prolonged examination (with a binocular) of the end of the comet's tail. Should you think the peculiar features which I have endeavoured to portray of sufficient interest to reproduce, the drawing is at your service. It is difficult to indicate truly features of this kind without exaggeration, if they are to catch the eye at all; but I am sure the exaggeration is very slight. The tail would seem to be about to end rather suddenly and with a broad end, when, from near the middle, shoots out, at a slight inclination to the general direction of the tail, a cleanly-shaded wisp.

It is surely unusual for such decided features

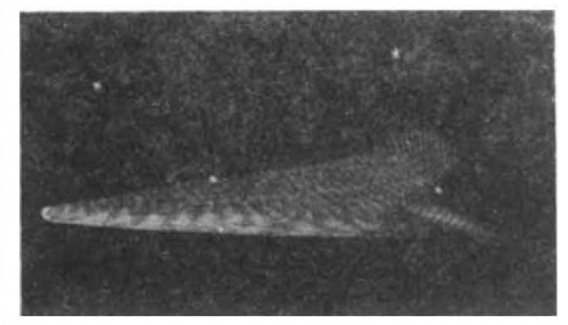

to present themselves at the very end of a comet's tail.

As a whole, the comet seems to have changed wonderfully little during the three weeks since I first saw it. Its change of place, also, is so moderate that, at this rate, there seems no reason why we should not see it for months yet. What if it should not vanish at all!

J. Hershcel.

Collingwood, October 23

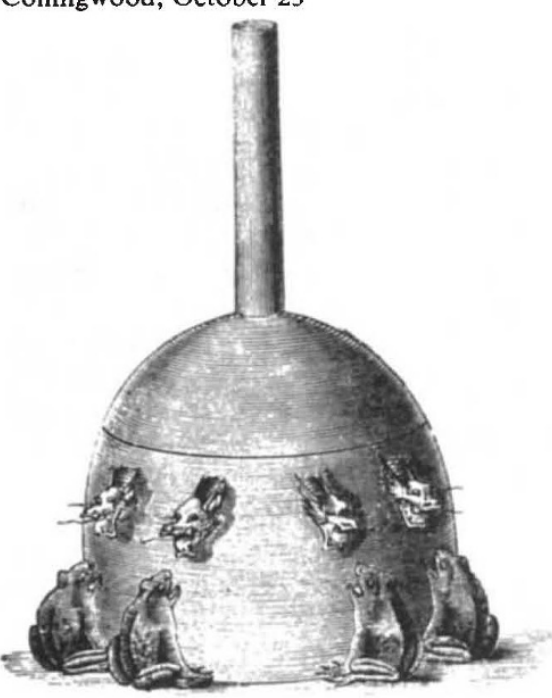

In a Chinese history called "Gokanjo", we find the following: "In the first year of Yoka (A.D. 136) a Chinese called Chioko invented a seismometer. This instrument consists of a sperically formed copper vessel (Fig.1), its diameter being 8 'shaku.' It is covered at its top. Its form resembles a wine bottle. Its outer part is ornamented with the figures of different kinds of birds and animals and old peculiar looking letters. In the inner part of this instrument a pillar is so placed that it can move in eight directions. Also in the inside of this bottle there is an arrangement by which some record of an earthquake is made according to the movement of the pillar. On the outside of the bottle there are eight dragon heads, each of which contains a ball. Underneath these heads there are eight frogs, so placed that they appear to watch the dragon's face, so that they are ready to receive the ball if it should be dropped. All the arrangements which cause the pillars when it moves to knock the ball out of the dragon's mouth are well hidden in the bottle. When an earthquake occurs and the bottle is shaken, the dragon instantly drops the ball, and the frog which receives it vibrates vigorously. Any one watching this instrument can easily observe earthquakes. With this arrangement, although one dragon may drop a ball, it is not necessary for the other seven dragons to drop their balls unless the movement has been in all directions; thus one can easily tell the direction of an earthquake. Once upon a time a dragon dropped its ball without any earthquake, and the people therefore thought that this instrument was of no use, but after two or three days a notice came saying that an earthquake had taken place in Rosei. Hearing of this, those who did not believe about the use of this instrument began to believe in it again. After this ingenious instrument had been invented by Chioko, the Chinese Government wisely appointed a secretary to make observations on earthquakes.'

We have here I think not only an account of an earthquake instrument which in principle is identical with many of our modern inventions, but the science has been conjoined with art. The record of the Chinese Government establishing a seismological bureau at a time when America was unknown, and half of Western Europe were living in the woods, is also interesting.

From Nature 26, 627; October 26, 1882. 